\title{
Follicular Dendritic Cell Sarcoma - A Case Report
}

\author{
Sagar Ramesh Bhalerao ${ }^{1}$, Aditya Shirish Adhav ${ }^{1}$, Rajnish Vasant Nagarkar ${ }^{1}$ \\ ${ }^{1}$ HCG Manavata Cancer Centre. Near Mylan Circle, Mumbai Naka, Nashik- 422044.
}

\section{Abstract}

Follicular dendritic cell sarcoma is an uncommon tumor that originates from follicular dendritic cells. The identification and diagnosis of FDCS is not as easy as it has close histological resemblance with non-Hodgkin lymphoma, sarcoma, melanoma, undifferentiated carcinomas, melanoma, and other dendritic or histiocytic cell disease. The definite diagnosis of FDCS can be established with the help of immunohistochemical investigation coupled with imaging modalities such as positron-emission tomography and computed tomography. We present a case of an elderly woman with a history of follicular dendritic tumor. The patient was primarily treated with CHOP chemotherapy in a different hospital. Upon initial investigation in our institution, disease progression was observed. The patient was treated with GEMTAX chemotherapy. The patient showed a partial response to therapy after three cycles of GEMTAX. However, disease progression was observed after six cycles of GEMTAX. The patient was switched to ESHAP, wherein after three cycles partial response to therapy was observed as per PET-CT scans. Despite the use of all three chemotherapy regimens, recurrence was observed. Systemic chemotherapy should be reserved for patients who failed in primary treatment or those with metastatic disease. However, multisite clinical trials should be developed to rationalize optimal treatment strategies for this uncommon disease.

Keywords: Follicular Dendritic Cell Sarcoma; Mesentery

Corresponding Author: Dr. Sagar Ramesh Bhalerao, HCG Manavata Cancer Centre. Near Mylan Circle, Mumbai Naka, Nashik- 422044.

Received: April 2019

Accepted: May 2019

\section{Introduction}

Dendritic cells are specialized immune cells that are involved in endocytosis and antigen presentation. They are classified as B $\square$ cell-associated dendritic cells or $\mathrm{T} \square$ cellassociated dendritic cells. Follicular dendritic cells are a type of B-cell dendritic cells that are located within the lymph follicles. Unlike other dendritic cells, follicular dendritic cells have no endocytosis or antigen presenting functions. Follicular dendritic cells originate from the mesenchyme and play a key role in the activation of B cells and maintenance of the lymph follicle environment within the lymph follicles. $^{[1]}$

Follicular dendritic cell sarcoma (FDCS) is also referred to as dendritic reticulum cell sarcoma. It was first described in 1986 by a team of researchers based on a series of four cases. FDCS was reported in the lymph nodes in all cases. However, only a few cases have been reported since then in the literature. In most cases, major sites of FDCS involve lymph nodes while nearly one-third of cases involve extranodal sites. ${ }^{[2]}$ Since the incidence of FDCS is low and that there is limited experience of the disease, it is often not considered during initial investigation or misdiagnosed.

FDCS is a very rare tumor that originates from follicular dendritic cells. FDCS is not easy to identify as it has similar histological similarities with sarcoma, non-Hodgkin lymphoma, undifferentiated carcinomas, melanoma, and other histiocytic cell or dendritic cell disease. ${ }^{[3]}$

Immunohistochemical investigations are required for the definite diagnosis of FDCs. Morphologically, FDCS is characterized by diffuse sheets, whorls, spindled to ovoid cells forming fascicles or nodules. In most cases, tumor tissue would present lymphoplasmacytic infiltration. ${ }^{[4]}$ Follicular dendritic cell differentiation markers are often expressed by tumor cells. Some of the markers include CD21, CD23, and CD35. Additionally, podoplanin, fascin, and clusterin are found positive during immunohistochemical investigation. ${ }^{[4]}$

Surgery has been considered as the standard therapy for patients presenting with local disease. Adjuvant chemotherapy has no significant impact on survival outcomes. ${ }^{[5]}$ In cases of multi-organ involvement or unresectable disease, chemotherapy may benefit. ${ }^{[6]}$ Till date, there is no standard or optimal chemotherapy regimen for this rare disease. Thus, there is a need to accumulate case reports, understand the pathophysiology of FDCS and determine an optimal treatment plan for such patients.

\section{Case Report}

A 61-year-old man reports to the out-patient department (OPD) with acute abdominal pain and generalized weakness. The patient was treated at a previous hospital for follicular dendritic tumor. The patient had received 5 cycles of 
chemotherapy with cyclophosphamide, doxorubicin, vincristine, and prednisone (CHOP) protocol. Reassessment of consecutive computed tomography (CT) scan showed progressive disease.

On initial examination at our OPD, the patient's vital signs were stable with no evidence for weight loss or fever. Physical examination revealed palpable, fixed tender mass in the left side of the abdomen extending from hypochondrium to mesogastrium on left. The laboratory data were normal. A positron-emission tomography (PET) scan was conducted initially. The scan revealed heterogeneously enhancing hypermetabolic well defined soft tissue mass in mesentery in the left lumbar region with necrotic areas and calcification foci within it. Multiple enhancing peri-lesional nodes were observed. Multiple hepatic deposits were observed. Multiple retro-peritoneal nodes with no abnormal metabolic activity were also observed [Figure 1].

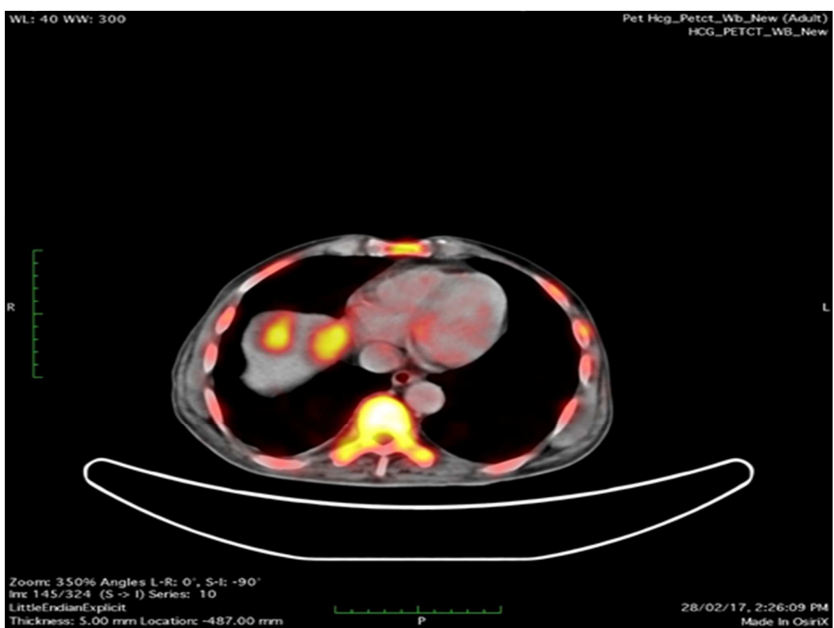

Figure 1: PET-CT Scan- Heterogeneously enhancing hypermetabolic well-defined soft tissue mass in mesentery in the left lumbar region with necrotic areas and calcification foci within it is observed.

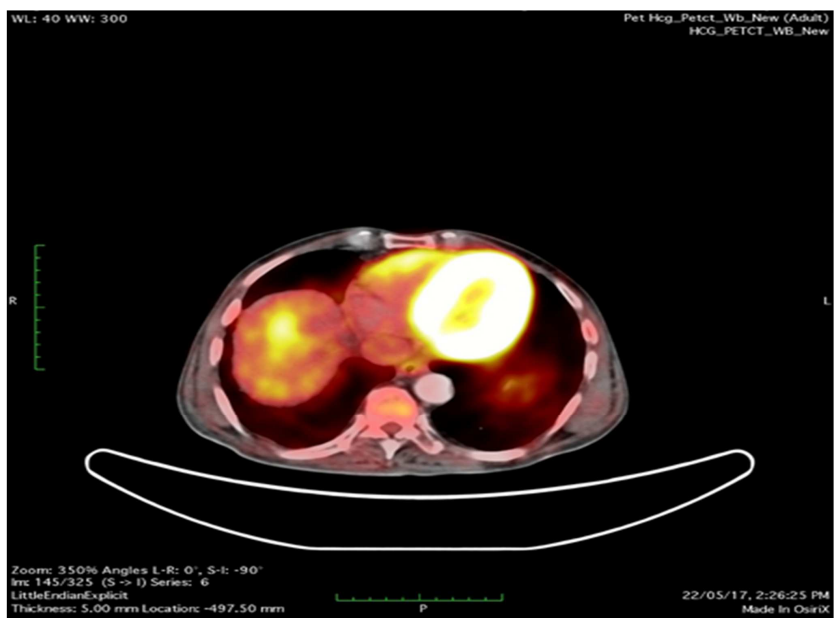

Figure 2: PET-CT Scan: Well-defined soft tissue mass in mesentery in the left lumbar region measuring $11.8 \times 8.1 \times 7.4$ cm is observed.

The patient was treated with gemcitabine/docetaxel (GEMTAX) chemotherapy.

A follow-up PET scan revealed well defined soft tissue mass in mesentery in the left lumbar region measuring $11.8 \mathrm{x} 8.1 \mathrm{x}$ $7.4 \mathrm{~cm}$ [Figure 2]. Multiple necrotic areas and calcification foci within the mass were observed. Fat planes with left psoas muscle, small and large bowel are maintained. Mild hepatomegaly with metastatic hepatic lesions was observed. Dilated bowel loops were observed within the abdominal cavity. However, features of intestinal obstruction were absent.

After 3 cycles of GEMTAX, the patient was evaluated using PET. As per the investigation, stable hypermetabolic soft tissue mass in mesentery in the left lumbar region was observed. The central necrosis was observed to have increased. However, hepatic metastatic lesions appeared to have reduced in size with resolution/reduction in metabolic activity for most of the lesions. Based on the overall radiological investigation, the patient appeared to have a partial response to therapy. The patient was treated with a total of six cycles of GEMTAX protocol. Follow-up investigation using PET revealed an increase in size and metabolic activity of metastatic hepatic lesions. Mild splenomegaly with increased metabolic activity was also observed [Figure 3]. The overall radiological investigation was suggestive of disease progression despite rigorous chemotherapy.

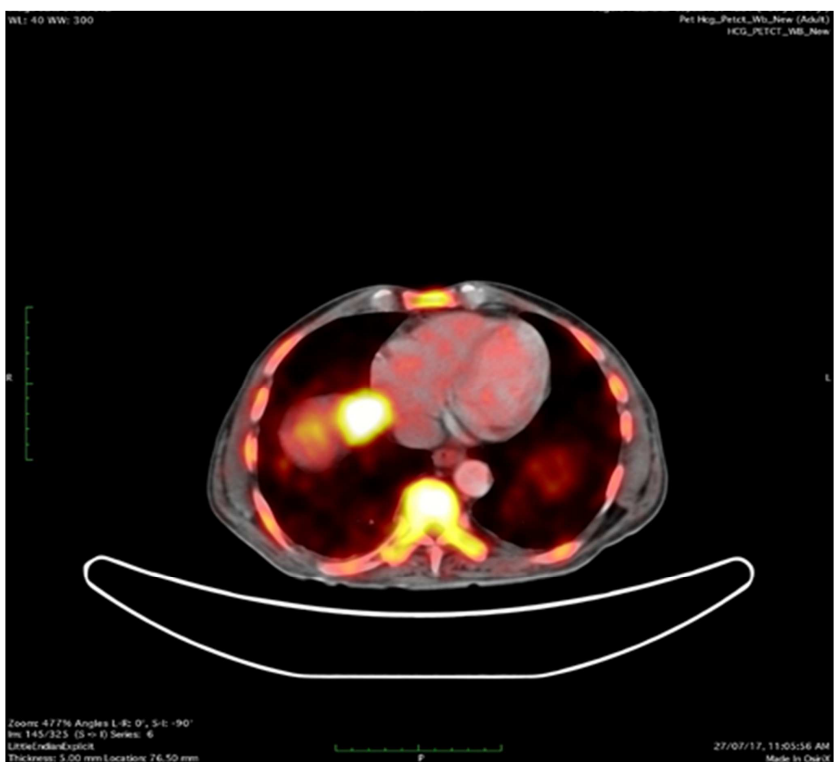

Figure 3: Follow-up PET-CT scan: An increase in size and metabolic activity of metastatic hepatic lesions. Mild splenomegaly with increased metabolic activity was also observed.

The patient was switched to etoposide, methylprednisolone, cytarabine \& cisplatin (ESHAP) chemotherapy. The patient was treated with a total of three cycles of ESHAP. Followup investigation using PET scan revealed a stable size of mesenteric soft tissue mass in the left lumbar region with central necrosis and reduced metabolic activity. Significant reduction in metabolic activity and mild reduction in the size of the metastatic hepatic lesions was observed. Mild reduction in the non-FDG avid perilesional and retroperitoneal nodes was observed (Fig. 4). The overall features are suggestive of a partial response to therapy. Unfortunately, the patient died after a few weeks. 


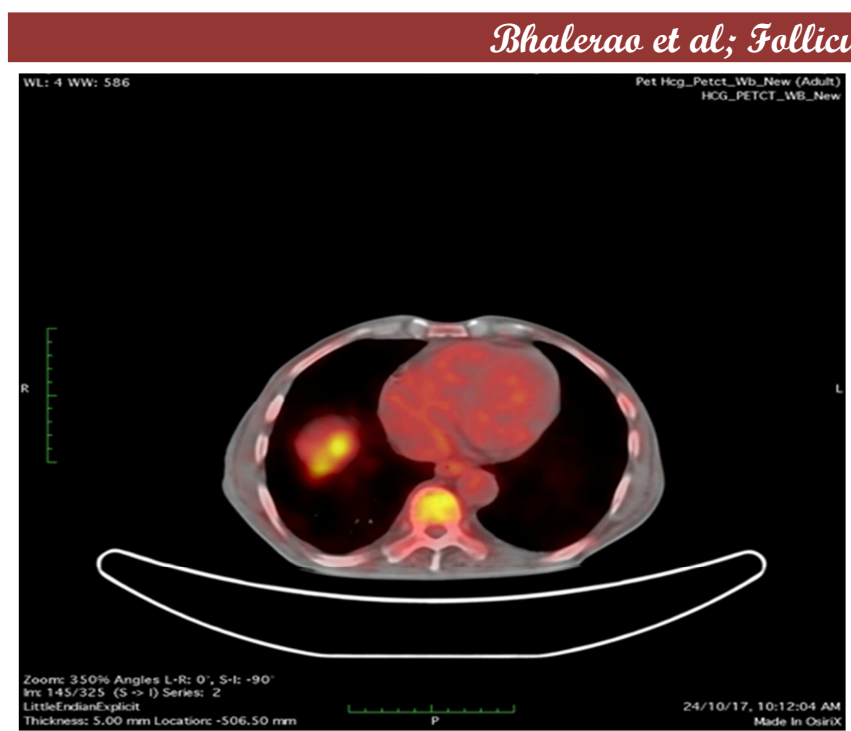

Figure 4: Follow-up PET-CT scan: Significant reduction in metabolic activity and mild reduction in the size of the metastatic hepatic lesions was observed. Mild reduction in the non-FDG avid perilesional and retroperitoneal nodes was observed.

\section{Discussion}

FDCS is an uncommon neoplasm that is known to have lowto-intermediate malignant potential. In patients with FDCS, slow-growing and painless masses would be observed without any systemic symptoms. ${ }^{[7]}$ However, patients with abdominal disease may present with abdominal pain as seen in our case.

FDCS are commonly in the peripheral lymph nodes, wherein the cervical nodes are the most common site. Some other sites affected by FDCS include retroperitoneal, mediastinal, and mesenteric area lymph nodes. ${ }^{[8]}$ In our case, heterogeneously enhancing hypermetabolic well defined soft tissue mass in mesentery in the left lumbar region was observed. In context to the diagnosis of FDCS, imaging modalities can be of great help. Imaging tools can help in assessing the extent of mass and staging. Ultrasound and CT are the preferred imaging modalities for the initial diagnosis of FDCS. ${ }^{[8]}$ In our case, PET scans helped in the initial assessment of the patient as well as follow-up evaluation to assess disease progression or response to therapy.

As per current literature, surgical resection is a preferred choice for FDCS while there is no standard chemotherapy or adjuvant therapy. The CHOP chemotherapy regimen has been widely used by oncologists, wherein some degree of response has been observed. In our case, the patient showed good initial response post-GEMTAX chemotherapy regimen. However, disease progression was noted after three cycles of GEMTAX regimen. ESHAP chemotherapy was used as a third-line chemotherapy treatment. The patient showed a partial response to therapy but died after several weeks.

\section{Conclusion}

To conclude, FDCS is an uncommon neoplasm. However, the frequency of FDCS has increased in the past few years. In patients presenting with lymph node enlargement, the disease should be kept in mind. It is difficult to provide effective treatment recommendations while surgical resection remains the mainstay treatment for localized disease. The possible roles for chemotherapy or radiotherapy remain unclear in such cases. Systemic chemotherapy should be reserved for patients who failed in primary treatment or those with metastatic disease. In our case, the use of CHOP, GEMTAX, and ESHAP chemotherapy regimen were used. However, partial response was observed with recurrence in all three treatment regimens. Multisite clinical trials can help understand and rationalize optimal treatment strategies for this uncommon tumor.

\section{Acknowledgements:}

I would like to thank Mr. Lyndon Fernandes for assisting us in writing.

\section{References}

1. Sasaki M, Izumi H, Yokoyama T, Kojima M, \& Hosono A. Follicular dendritic cell sarcoma treated with a variety of chemotherapy. Hematological Oncology 2017;35(4):905-908.

2. Ma Y, Sun, J, Yang C, Yuan D, \& Liu, J. Follicular dendritic cell sarcoma: two rare cases and a brief review of the literature. OncoTargets and Therapy 2015;8, 1823-1830.

3. Sasaki M, Izumi H, Yokoyama T, Kojima M, \& Hosono A. Follicular dendritic cell sarcoma treated with a variety of chemotherapy. Hematological Oncology 2017; 35(4), 905-908.

4. Wu A, Pullarkat S. Follicular Dendritic Cell Sarcoma. Arch Pathol Lab Med. 2016;140(2):186-90.

5. Saygin C, Uzunaslan D, Ozguroglu M, Senocak M, Tuzuner N. Dendritic cell sarcoma: a pooled analysis including 462 cases with presentation of our case series. Crit Rev Oncol Hematol. 2013;88(2):253-71.

6. Soriano AO, Thompson MA, Admirand JH, Fayad LE, Rodriguez AM, Romaguera JE, Hagemeister FB, Pro B. Follicular dendritic cell sarcoma: a report of 14 cases and a review of the literature. Am J Hematol. 2007;82(8):725-8.

7. Choi BS, Baek JH, Shin YM, Kim JH, Kim HW, Lee SJ, \& Cha HJ. Follicular Dendritic Cell Sarcoma: A Case Report and Review of the Literature. Cancer Research and Treatment: Official Journal of Korean Cancer Association, 2010;42(2), 121-124.

8. Chang, YC, Chau IY, Yeh YC, \& Chau GY. Small intestine follicular dendritic cell sarcoma with liver metastasis: A case report. Medicine, 2017;96(31), e7261.

Copyright: ( $)$ the author(s), publisher. Academia Anatomica International is an Official Publication of "Society for Health Care \& Research Development". It is an open-access article distributed under the terms of the Creative Commons Attribution Non-Commercial License, which permits unrestricted non-commercial use, distribution, and reproduction in any medium, provided the original work is properly cited.

How to cite this article: Bhalerao SR, Adhav AS, Nagarkar RV. Follicular Dendritic Cell Sarcoma- A Case Report. Acad. Anat. Int. 2019;5(1):93-95.

DOI: dx.doi.org/10.21276/aanat.2019.5.1.22 